\title{
Paxillin expression is closely linked to the pathogenesis, progression and prognosis of gastric carcinomas
}

\author{
LI-JUN XIAO ${ }^{1}$, EN-HONG ZHAO ${ }^{2}$, SHUANG ZHAO ${ }^{1}$, XIN ZHENG $^{2}$, HUA-CHUAN ZHENG $^{1}$, \\ YASUO TAKANO ${ }^{3}$ and HONG-RU SONG ${ }^{1}$
}

\author{
${ }^{1}$ Department of Immunology, Chengde Medical University, Chengde, Hebei 067000; \\ ${ }^{2}$ Third Surgical Department, Affiliated Hospital of Chengde Medical University, Chengde, Hebei 067000, P.R. China; \\ ${ }^{3}$ Clinical Cancer Institute, Kanagawa Cancer Center, Yokohama, Kanagawa 250-0134, Japan
}

Received March 25, 2013; Accepted October 7, 2013

DOI: $10.3892 / 01.2013 .1686$

\begin{abstract}
Paxillin encodes a focal adhesion-associated protein and is involved in the progression and aggressive phenotypes of malignancies through its interactions with the actin cytoskeleton and key signal transduction oncogenes. The present study aimed to investigate the clinicopathological and prognostic significance of paxillin in gastric cancer. The expression of paxillin was evaluated using tissue microarrays of gastric adjacent non-cancerous mucosa, adenoma and carcinoma specimens by immunohistochemistry. Paxillin expression was compared against clinicopathological parameters and the survival time of the patients. Paxillin was highly expressed in gastric adenoma compared with that in non-neoplastic mucosa and carcinoma $(\mathrm{P}<0.05)$. Paxillin expression was lower in the younger carcinoma patients compared with that in the elder carcinoma patients $(\mathrm{P}<0.05)$. Paxillin expression was negatively correlated with tumor size, depth of invasion and lymph node metastasis, but not with patient gender, lymphatic or venous invasion, or TNM staging $(\mathrm{P}>0.05)$. Higher paxillin expression was observed in intestinal-type compared with diffuse-type carcinoma $(\mathrm{P}<0.05)$. Kaplan-Meier analysis indicated a positive association between paxillin expression and cumulative survival rate in all, advanced and intestinal-type carcinoma patients $(\mathrm{P}<0.05)$. Multivariate analysis using the Cox proportional hazards model indicated that patient age, depth of invasion, lymphatic invasion, lymph node metastasis, TNM staging and Lauren classification were independent prognostic factors for all gastric carcinomas $(\mathrm{P}<0.05)$. Aberrant
\end{abstract}

Correspondence to: Professor Hong-Ru Song, Department of Immunology, Chengde Medical University, Shang Er Dao He Zi, Chengde, Hebei 067000, P.R.China

E-mail: songhongru@163.com

Key words: gastric carcinoma, tumorgenesis, paxillin, clinicopathological behaviors, prognosis paxillin expression may be involved in the growth, invasion, metastasis and differentiation of gastric carcinoma. Altered paxillin expression may, therefore, be employed as an indicator of pathobiological behaviors and prognosis of gastric carcinomas.

\section{Introduction}

Despite a global decline in the incidence and mortality of gastric cancer in the last 60 years, it remains the fourth most common and second most frequent cause of cancer-related mortality. Gastric cancer continues to be a major health concern due to the slow decrease in incidence in Asia and high mortality from diagnosed gastric carcinomas in the West, despite the advanced diagnostic and operative techniques that are commonly used in clinical practice $(1,2)$. An increased understanding of the changes that occur in gene expression in gastric cancer, particularly the identification of novel biomarkers for cancer diagnosis and novel targets for treatment, is required for the improvement of diagnosis, treatment and prevention.

Paxillin is a focal adhesion-associated, phosphotyrosine-containing 68-kDa adaptor protein discovered in 1990 by Turner et al (3). Paxillin contains a number of motifs that mediate protein-protein interactions, including C-terminal LIM domains resembling a double zinc-finger domain, N-terminal LD motifs, SH3 and SH2 domain-binding sites, whose motifs serve as docking sites for cytoskeletal proteins, tyrosine kinases, serine/threonine kinases, GTPase activating proteins and other adaptor proteins that recruit additional enzymes into complex with paxillin (4). Multiple tyrosine, serine and threonine phosphorylation sites exist throughout the paxillin molecule, and are targeted by a diverse array of kinases that are activated in response to various adhesion stimuli and growth factors (PDGF, EGF and IL-3). These include p21-activated kinase, FAK-Src, receptor for activated $\mathrm{C}$ kinase 1 , c-Jun $\mathrm{N}$-terminal kinase, extracellular-signal-regulated kinase, p38 mitogen-activated protein kinase, cyclin-dependent kinase 5 and c-Abl. Paxillin is tyrosine-phosphorylated upon integrin engagement or growth factor stimulation, creating binding sites for the Crk adapter protein $(5,6)$. Thus, paxillin may be involved in signal 


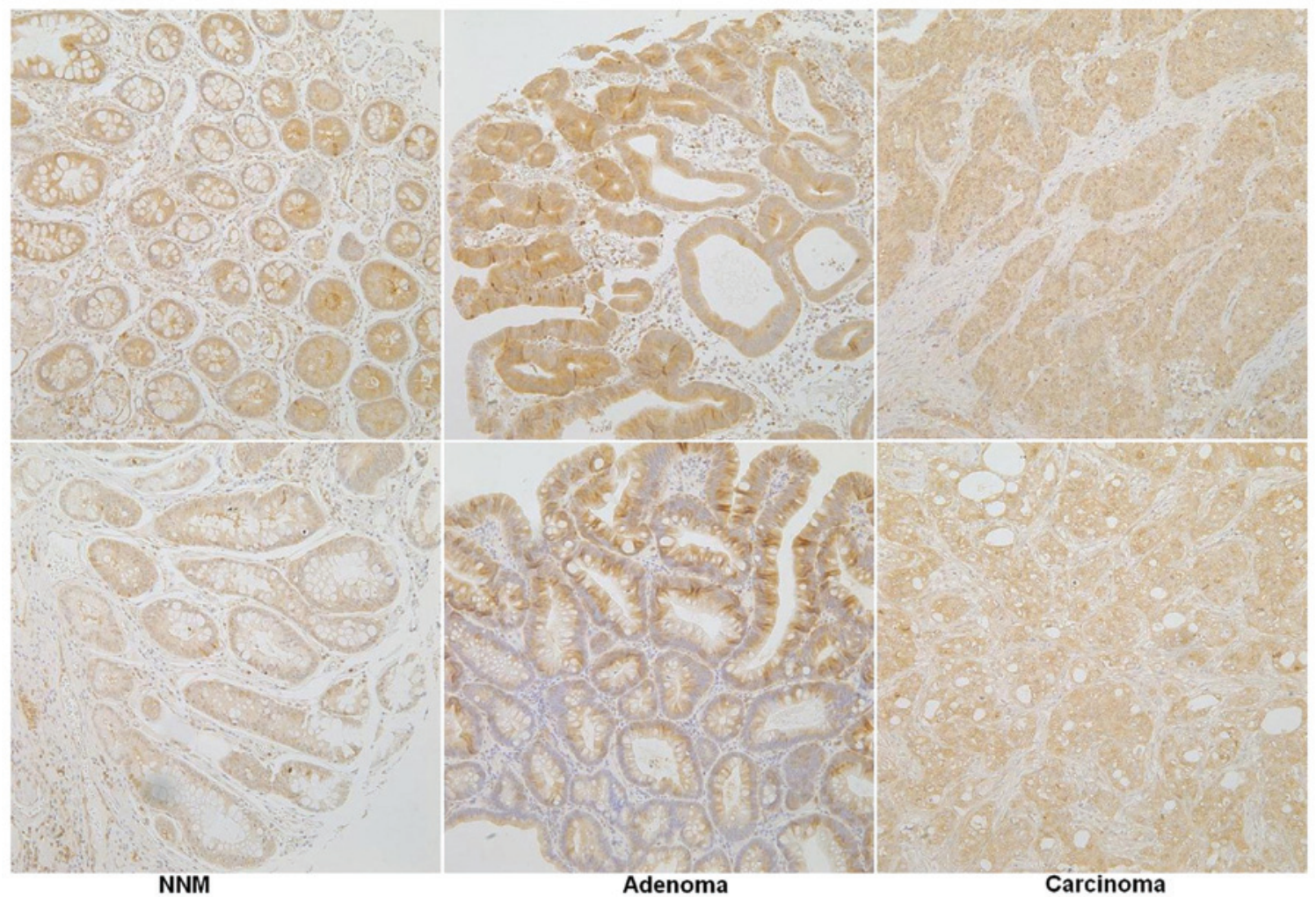

Figure 1. Immunohistochemical staining of gastric tissue samples showing the expression of paxillin. Strong positivity of paxillin was localized in the cytoplasm of gastric non-neoplasmic mucosa (NNM), adenoma and carcinoma (magnification, $\mathrm{x} 400$ ).

transduction, regulation of cell morphology and the recruitment of structural and signaling molecules to focal adhesions to control cell spread and migration $(7,8)$.

Previous studies have demonstrated that paxillin was overexpressed in esophageal squamous cell carcinoma, lung carcinoma, breast cancer and prostate cancer (9-13). In breast cancer, it has been found that the overexpression of paxillin may represent a useful prognosticator and be employed to predict the clinical response to chemotherapy $(12,14)$. To better understand the clinicopathological and prognostic significance of paxillin, we observed its expression in gastric non-neoplastic mucosa, adenoma and carcinoma using a combination of tissue microarray and immunohistochemistry. Paxillin expression was compared with the clinicopathological and prognostic features of gastric cancer.

\section{Materials and methods}

Patients. This retrospective study was carried out using curatively resected specimens of gastric cancer $(n=392)$ and adjacent non-neoplastic mucosa $(n=197)$ collected at Toyama University Hospital (Toyama, Japan) from 1993 to 2006. The adenoma samples were resected from endoscopic biopsy at Toyama University Hospital from 1997 to 2008. The patients with gastric carcinomas were 120 males and 272 females (38-88 years; mean, 66.7 years). Archival materials were obtained from the Department of Pathology of Toyama University Hospital . In 151 cases, tumor development was accompanied by lymph node metastasis. None of the patients underwent chemotherapy, radiotherapy and adjuvant treatment prior to surgery. All patients were followed up by consulting their case documents and by telephone.

Pathology. All tissues were fixed in $10 \%$ neutralized formalin, embedded in paraffin, cut into $4-\mu \mathrm{m}$ sections and stained with hematoxylin and eosin (H\&E) in order to confirm the histological diagnosis and microscopic characteristics of the specimens. The staging for each gastric carcinoma was evaluated according to the Union for International Cancer Control system, which indicates the extent of tumor spread (15). Histological architecture was defined using the Lauren classification $(16,17)$. The tumor size, depth of invasion, lymphatic and venous invasion, and lymph node metastasis of tumors were also determined.

Tissue microarray (TMA). From H\&E-stained sections of the tumor cases, representative areas of solid tumor were selected for sampling and 2-mm diameter tissue cores per donor block were punched out and transferred to a recipient block with a maximum of 48 cores using a tissue microarrayer (KIN-1; Azumaya, Tokyo, Japan). Sections $(4-\mu \mathrm{m})$ were consecutively cut from the microarrays and transferred to poly-lysine-coated glass slides.

Immunohistochemistry. Serial sections of TMA were deparaffinized with xylene, rehydrated with alcohol, and subjected to immunohistochemical staining with intermittent microwave radiation, as previously described (18). Rabbit anti-human paxillin antibody (Epitomics, Inc., Burlingame, CA, USA) was used at 1:100 dilution to detect the respective proteins, with 
Table I. Paxillin expression in gastric carcinomas.

\begin{tabular}{lrrrrrr}
\hline & & \multicolumn{4}{c}{ Paxillin expression } & \\
\cline { 3 - 6 } Groups & $\mathrm{n}$ & - & + & ++ & +++ & PR (\%) \\
\hline Non-cancerous mucosa & 197 & 70 & 91 & 26 & 10 & 64.5 \\
Adenoma & 67 & 7 & 21 & 28 & 11 & $92.3^{\text {a }}$ \\
Carcinoma & 392 & 130 & 164 & 59 & 39 & 66.8 \\
\hline
\end{tabular}

${ }^{a} \mathrm{P}<0.001$, compared with non-cancerous mucosa or carcinoma. $\mathrm{PR}$, positive rate.

Table II. Correlation between paxillin expression and clinicopathological features of gastric carcinomas.

\begin{tabular}{|c|c|c|c|c|c|c|c|}
\hline \multirow[b]{2}{*}{ Clinicopathological features } & \multirow[b]{2}{*}{$\mathrm{n}$} & \multicolumn{4}{|c|}{ Paxillin expression } & \multirow[b]{2}{*}{ PR $(\%)$} & \multirow[b]{2}{*}{ P-value } \\
\hline & & - & + & ++ & +++ & & \\
\hline Age (years) & & & & & & & 0.027 \\
\hline$<65$ & 156 & 56 & 68 & 19 & 13 & 64.1 & \\
\hline$\geq 65$ & 236 & 74 & 96 & 40 & 26 & 68.6 & \\
\hline Gender & & & & & & & 0.060 \\
\hline Male & 272 & 89 & 105 & 46 & 32 & 67.3 & \\
\hline Female & 120 & 41 & 59 & 13 & 7 & 65.8 & \\
\hline Tumor size $(\mathrm{cm})$ & & & & & & & 0.001 \\
\hline$<4$ & 204 & 58 & 80 & 40 & 26 & 71.6 & \\
\hline$\geq 4$ & 188 & 72 & 84 & 19 & 13 & 61.7 & \\
\hline Depth of invasion & & & & & & & $<0.001$ \\
\hline $\mathrm{T}_{\mathrm{is}^{-} 1}$ & 200 & 59 & 85 & 35 & 21 & 70.5 & \\
\hline $\mathrm{T}_{2^{-} 4}$ & 192 & 71 & 79 & 24 & 18 & 63.0 & \\
\hline Lymphatic invasion & & & & & & & 0.799 \\
\hline- & 250 & 80 & 111 & 32 & 27 & 68.0 & \\
\hline+ & 142 & 50 & 53 & 27 & 12 & 64.8 & \\
\hline \multicolumn{8}{|l|}{ Venous invasion } \\
\hline- & 335 & 113 & 142 & 48 & 32 & 66.3 & 0.287 \\
\hline+ & 57 & 17 & 22 & 11 & 7 & 70.2 & \\
\hline \multicolumn{8}{|l|}{ Lymph node metastasis } \\
\hline- & 241 & 70 & 102 & 39 & 30 & 71.0 & 0.006 \\
\hline+ & 151 & 60 & 62 & 20 & 9 & 60.3 & \\
\hline UICC staging & & & & & & & 0.352 \\
\hline 0 -I & 215 & 68 & 89 & 36 & 22 & 68.4 & \\
\hline II-IV & 177 & 62 & 75 & 23 & 17 & 65.0 & \\
\hline Lauren classification & & & & & & & $<0.001$ \\
\hline Intestinal type & 210 & 46 & 98 & 36 & 30 & 78.1 & \\
\hline Diffuse type & 172 & 80 & 62 & 22 & 8 & 53.5 & \\
\hline
\end{tabular}

PR, positive rate; $\mathrm{T}_{\mathrm{is}}$, carcinoma in situ; $\mathrm{T}_{1}$, lamina propria and submucosa; $\mathrm{T}_{2}$, muscularis propria and subserosa; $\mathrm{T}_{3}$, exposure to serosa; $\mathrm{T}_{4}$, invasion into serosa; UICC, Union for International Cancer Control.

anti-rabbit Envison-PO (Dako, Carpinteria, CA, USA) as the secondary antibody. Binding was visualized with 3,3'-diaminobenzidine and counterstaining with Mayer's hematoxylin was performed to aid orientation. Omission of the primary antibody was used as a negative control.
Immunoreactivity for paxillin showed a cytoplasmic pattern (Fig. 1). One hundred cells were randomly selected and counted from five representative fields of each section, blindly, by three independent observers (L.J. Xiao and H.C. Zheng). The inconsistent data were confirmed by both observers until 
Table III. Multivariate analysis of clinicopathological variables for survival with gastric carcinomas.

\begin{tabular}{llc}
\hline $\begin{array}{l}\text { Clinicopathological } \\
\text { parameters }\end{array}$ & $\begin{array}{c}\text { Relative risk } \\
(95 \% \mathrm{CI})\end{array}$ & P-value \\
\hline Age ( $\geq 65$ years) & $1.902(1.254-2.883)$ & 0.002 \\
Gender (male) & $1.212(0.750-1.959)$ & 0.432 \\
Tumor size $(\geq 4 \mathrm{~cm})$ & $1.285(0.771-2.141)$ & 0.336 \\
Depth of invasion $\left(\mathrm{T}_{2-4}\right)$ & $5.979(2.084-17.152)$ & 0.001 \\
Lymphatic invasion $(+)$ & $1.995(1.201-3.313)$ & 0.008 \\
Venous invasion $(+)$ & $1.202(0.751-1.922)$ & 0.444 \\
Lymph node metastasis $(+)$ & $2.932(1.535-5.602)$ & 0.001 \\
TNM staging (III-IV) & $0.341(0.119-0.974)$ & 0.045 \\
Lauren classification & & \\
(diffuse type) & $2.235(1.396-3.577)$ & 0.001 \\
Paxillin expression $(+\sim+++)$ & $0.714(0.475-1.073)$ & 0.105 \\
\hline
\end{tabular}

$\mathrm{CI}$, confidence interval; $\mathrm{T}_{2}$, muscularis propria and subserosa; $\mathrm{T}_{3}$, exposure to serosa; $\mathrm{T}_{4}$, invasion into serosa; TNM, tumor-node-metastasis .

final agreements were reached. The expression positivity was graded and counted as follows: $0=0 \% ; 1=1-49 \% ; 2=50-74 \%$; and $3 \geq 75 \%$. The staining intensity score was graded as follows: 1 = weak; 2 = intermediate; and $3=$ strong. The scores for paxillin positivity and staining intensity were multiplied to obtain a final score, which determined their expression as $-=0 ;+=1-2 ;++=3-4$; or $+++=6-9$.

Statistical analysis. Statistical evaluation was performed using Spearman's rank correlation test. Kaplan-Meier survival plots were generated and comparisons between survival curves were made with the log-rank test. Cox proportional hazards model was employed for multivariate analysis. SPSS $17.0 \mathrm{soft}-$ ware (SPSS, Inc., Chicago, IL, USA) was applied to analyze all data, and $\mathrm{P}<0.05$ was considered to indicate a statistically significant difference.

\section{Results}

As indicated in Fig. 1, paxillin was positively expressed in the cytoplasm of gastric superficial epithelium, intestinal metaplasia, adenoma and carcinoma. The levels of paxillin expression was detected in gastric non-neoplastic mucosa $(64.5 \%, 127 / 197)$, adenoma $(92.3 \%, 60 / 67)$ and carcinoma $(66.8 \%, 262 / 392)$, respectively. The expression of paxillin was significantly more highly expressed in gastric adenoma than in non-neoplastic mucosa and carcinoma $(\mathrm{P}<0.05$, Table I). As shown in Table II, paxillin expression was negatively correlated with tumor size, depth of invasion, and lymph node metastasis, but not with gender, lymphatic or venous invasion, or TNM staging $(\mathrm{P}>0.05)$. Paxillin expression was higher in the elder carcinoma patients than in the younger carcinoma patients $(\mathrm{P}<0.05)$. There was higher paxillin expression in intestinal- compared with diffuse-type carcinoma $(\mathrm{P}<0.05)$.

Follow-up information was available on 392 of the gastric carcinoma patients for periods ranging from 0.2 months to 121 months (mean, 70.4 months). Fig. 2 shows survival curves
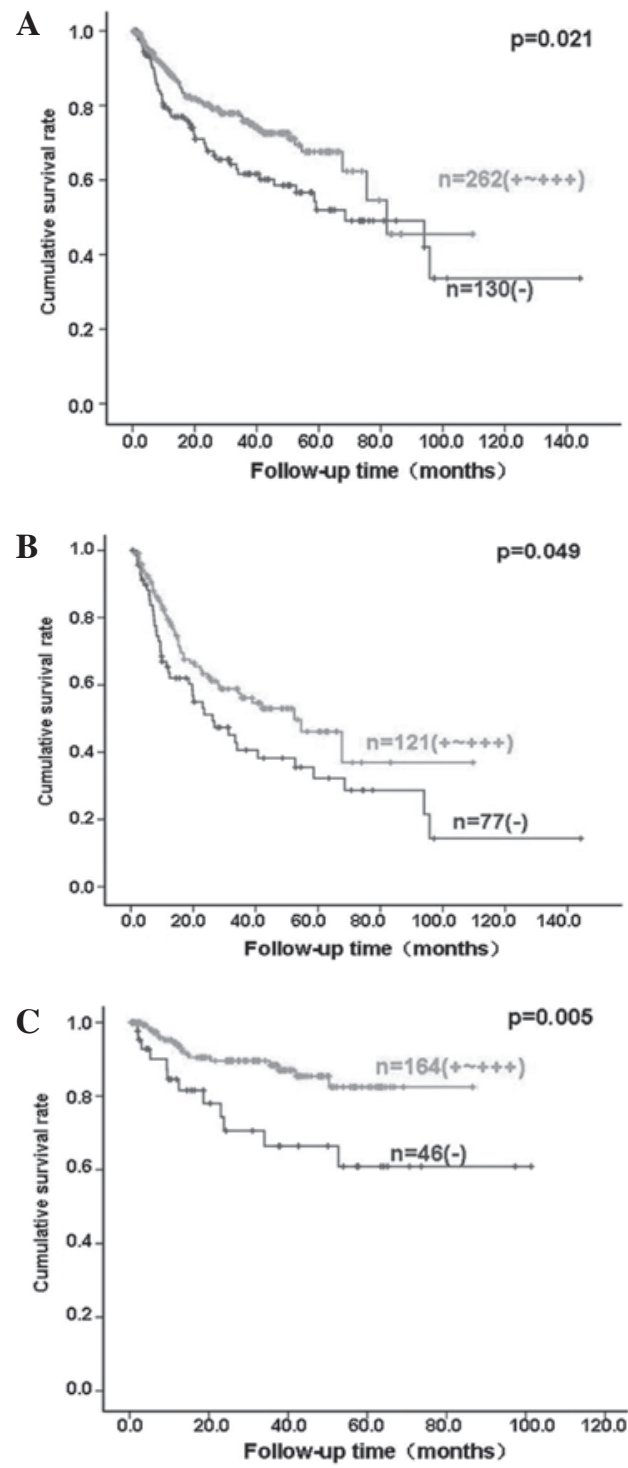

Figure 2. Prognostic significance of paxillin expression in patients with gastric cancer. Kaplan-Meier curves for cumulative survival rate of patients with (A) all, (B) advanced and (C) intestinal-type gastric carcinomas according to paxillin expression status.

stratified according to paxillin expression. Univariate analyses using the Kaplan-Meier method indicated a higher cumulative survival rate in all, advanced and intestinal-type carcinoma patients with weak, moderate and strong paxillin expression than in those without paxillin expression $(\mathrm{P}<0.05)$. Multivariate analysis using the Cox proportional hazards model indicated that patient age, depth of invasion, lymphatic invasion, lymph node metastasis, TNM staging and Lauren classification $(\mathrm{P}<0.05)$, but not patient gender, tumor size, venous invasion or paxillin expression $(\mathrm{P}>0.05)$, were independent prognostic factors for all gastric carcinomas (Table III).

\section{Discussion}

Paxillin is a cytoskeletal protein that was recently identified as a component of focal adhesions and links between F-actin and integrin (19). In the present study, the cytoplasmic expression pattern of paxillin was observed in the gastric non-neoplastic 
epithelial cells, adenomas and adenocarcinomas. Statistically, paxillin expression was increased in gastric adenoma in comparison with that in the non-neoplastic mucosa and carcinoma. The adenoma can progress into and be incorporated with gastric well-differentiated carcinoma when it grows larger and de novo carcinogenesis is well understood, particularly in diffuse-type gastric carcinomas (20). These findings suggested that aberrant paxillin expression may be involved in the progression from gastric adenoma to adenocarcinoma. Higher paxillin expression in adenoma and intestinal-type carcinoma indicated that paxillin overexpression may be closely linked to the intestinal carcinogenic pathway of gastric cancer.

Cai et al (21) found that paxillin mRNA expression levels were significantly correlated with the differentiation degree, depth of invasion and lymph node metastasis of esophageal carcinoma. A previous study indicated that paxillin expression was correlated with distant metastasis and clinical stage of salivary adenoid cystic carcinoma (22). Li et al (23) documented that positive paxillin expression was significantly associated with low differentiation, with the presence of portal vein thrombosis, and with extra-hepatic metastasis of hepatocellular cell carcinoma. Li et al (24) found that paxillin positivity in human gastric cancer was associated with tumor stage, and siRNA targeting paxillin decreased the phosphorylation of paxillin (tyr118) and the invasiveness of AGS cells significantly as compared with controls. Previously, it was identified that overexpression of wild-type paxillin plasmids promoted cell proliferation and also enhanced migration, invasive capacity and metastasis of the colorectal cancer cells (25). However, paxillin expression was negatively correlated with tumor size, depth of invasion and lymph node metastasis of gastric cancer in the present study. The contradictory phenomena should be further investigated in the future.

Although all types of gastric cancer are malignant tumors that originate from the same gastric epithelium, the morphological features of the cancers vary substantially in individual patients. According to Lauren classification, gastric intestinal-type carcinoma is characterized by cohesive carcinoma cells that form gland-like tubular structures, such as well- and moderately differentiated carcinoma; while cell cohesion is less apparent or absent in diffuse-type carcinoma, such as poorly differentiated or signet ring cell carcinoma $(16,17)$. Our findings demonstrated that paxillin was more highly expressed with a higher incidence in intestinal-type gastric cancer, which is presumed to arise from preceding dysplastic lesions, than diffuse-type ones, which evolve without any precedent dysplastic changes, indicating that distinct paxillin expression underlies the molecular mechanisms for the differentiation of intestinal- and diffuse-type carcinomas.

To date, there have been several studies describing the prognostic significance of paxillin expression in malignancies $(11,12,14,26)$. In the present study, for the first time, we analyzed the correlation between paxillin expression and the survival rate of 392 patients with gastric carcinoma. The results revealed a close association between its overexpression and favorable survival. When stratified according to the depth of invasion, the significant correlation disappeared in the early gastric cancers, but not in the advanced ones, indicating that the association between paxillin expression and prognosis depends on the depth of invasion. The multivariate analysis demonstrated that patient age, depth of invasion, lymphatic invasion, lymph node metastasis, TNM staging and Lauren classification, but not patient gender, tumor size, venous invasion or paxillin expression, were independent prognostic factors for all gastric carcinomas. These findings suggested that paxillin expression is a good indicator for the favorable prognosis of gastric carcinoma patients, albeit it is not independent. By contrast, Li et al (11) found that no correlation occurred between expression of paxillin and patient survival of these patients with esophageal cancer. Zuo et al (26) found that paxillin expression was closely correlated with the prognosis of non-small cell lung carcinoma.

In conclusion, aberrant paxillin expression may be important in the malignant transformation of gastric epithelial cells. Its reduced expression was closely correlated with growth, invasion, metastasis and a worse prognosis of gastric carcinomas. Its expression may be employed to differentiate between the intestinal- and diffuse-type carcinomas. It was considered as a promising marker to indicate the pathobiological behaviors and prognosis of gastric carcinomas.

\section{Acknowledgements}

This study was supported by Shenyang Science and Technology Grant (F11-264-1-10; F12-277-1-01); Liaoning Science and Technology Grant; Natural Scientific Foundation of China (81172371) and Grant-in aid for Scientific Research from the Ministry of Education, Culture, Sports and Technology of Japan (23659958).

\section{References}

1. Rivera F, Vega-Villegas ME and López-Brea MF: Chemotherapy of advanced gastric cancer. Cancer Treat Rev 33: 315-324, 2007.

2. Kelley JR and Duggan JM: Gastric cancer epidemiology and risk factors. J Clin Epidemiol 56: 1-9, 2003.

3. Turner CE, Glenney JR Jr and Burridge K: Paxillin: a new vinculin-binding protein present in focal adhesions. J Cell Biol 111: 1059-1068, 1990.

4. Deakin NO and Turner CE: Paxillin comes of age. J Cell Sci 121: 2435-2444, 2008.

5. Schaller MD: Paxillin: a focal adhesion-associated adaptor protein. Oncogene 20: 6459-6472, 2001.

6. Brown MC and Turner CE: Paxillin: adapting to change. Physiol Rev 84: 1315-1339, 2004.

7. Sattler M, Pisick E, Morrison PT and Salgia R: Role of the cytoskeletal protein paxillin in oncogenesis. Crit Rev Oncog 11: 63-76, 2000.

8. Huang C, Jacobson K and Schaller MD: A role for JNK-paxillin signaling in cell migration. Cell Cycle 3: 4-6, 2004.

9. Tremblay L, Hauck W, Aprikian AG, Begin LR, Chapdelaine A and Chevalier S: Focal adhesion kinase (pp125FAK) expression, activation and association with paxillin and p50CSK in human metastatic prostate carcinoma. Int J Cancer 68: 164-171, 1996.

10. Jagadeeswaran R, Surawska H, Krishnaswamy S, Janamanchi V, Mackinnon AC, Seiwert TY, Loganathan S, Kanteti R, Reichman T, Nallasura V, et al: Paxillin is a target for somatic mutations in lung cancer: implications for cell growth and invasion. Cancer Res 68: 132-142, 2008.

11. Li BZ, Lei W, Zhang CY, Zhou F, Li N, Shi SS, Feng XL, Chen ZL, Hang J, Qiu B, et al: Increased expression of paxillin is found in human oesophageal squamous cell carcinoma: a tissue microarray study. J Int Med Res 36: 273-278, 2008.

12. Short SM, Yoder BJ, Tarr SM, Prescott NL, Laniauskas S, Coleman KA, Downs-Kelly E, Pettay JD, Choueiri TK, Crowe JP, et al: The expression of the cytoskeletal focal adhesion protein paxillin in breast cancer correlates with HER2 overexpression and may help predict response to chemotherapy: a retrospective immunohistochemical study. Breast J 13: 130-139, 2007. 
13. Mackinnon AC, Tretiakova M, Henderson L, Mehta RG, Yan BC, Joseph L, Krausz T, Husain AN, Reid ME and Salgia R: Paxillin expression and amplification in early lung lesions of high-risk patients, lung adenocarcinoma and metastatic disease. J Clin Pathol 64: 16-24, 2011.

14. Scibelli A, d'Angelo D, Pelagalli A, Tafuri S, Avallone L, Della Morte R and Staiano N: Expression levels of the focal adhesion-associated proteins paxillin and p130CAS in canine and feline mammary tumors. Vet Res 34: 193-202, 2003.

15. Sobin LH and Wittekind CH: TNM Classification of malignant tumors, 6th edn. New Jersey: John Wiley \& Sons, Hoboken, 2002.

16. Zheng H, Takahashi H, Murai Y, Cui Z, Nomoto K, Miwa S, Tsuneyama K and Takano Y: Pathobiological characteristics of intestinal and diffuse-type gastric carcinoma in Japan: an immunostaining study on the tissue microarray. J Clin Pathol 60: 273-277, 2007.

17. Zheng HC, Li XH, Hara T, Masuda S, Yang XH, Guan YF and Takano Y: Mixed-type gastric carcinomas exhibit more aggressive features and indicate the histogenesis of carcinomas. Virchows Arch 452: 525-534, 2008.

18. Kumada T, Tsuneyama K, Hatta H, Ishizawa S and Takano Y: Improved 1-h rapid immunostaining method using intermittent microwave irradiation: practicability based on 5 years application in Toyama Medical and Pharmaceutical University Hospital. Mod Pathol 17: 1141-1149, 2004.

19. Chen J and Gallo KA: MLK3 regulates paxillin phosphorylation in chemokine- mediated breast cancer cell migration and invasion to drive metastasis. Cancer Res 72: 4130-4140, 2012.
20. Zheng HC, Tsuneyama K, Takahashi H, Miwa S, Sugiyama T, Popivanova BK, Fujii C, Nomoto K, Mukaida N and Takano Y: Aberrant Pim-3 expression is involved in gastric adenoma-adenocarcinoma sequence and cancer progression. J Cancer Res Clin Oncol 134: 481-488, 2008.

21. Cai HX, Yang LC, Song XH, Liu ZR, Chen YB and Dong GK: Expression of paxillin and FAK mRNA and the related clinical significance in esophageal carcinoma. Mol Med Rep 5: 469-472, 2012.

22. Shi J, Wang S, Zhao E, Shi L, Xu X and Fang M: Paxillin expression levels are correlated with clinical stage and metastasis in salivary adenoid cystic carcinoma. J Oral Pathol Med 39: 548-551, 2010.

23. Li HG, Xie DR, Shen XM, Li HH, Zeng H and Zeng YJ: Clinicopathological significance of expression of paxillin, syndecan-1 and EMMPRIN in hepatocellular carcinoma. World J Gastroenterol 11: 1445-1451, 2005.

24. Li D, Ding J, Wang X, Wang C and Wu T: Fibronectin promotes tyrosine phosphorylation of paxillin and cell invasiveness in the gastric cancer cell line AGS. Tumori 95: 769-779, 2009.

25. Jun Q, Zhiwei W, Lilin M, Jing $K$ and Qichao N: Effects of paxillin on HCT-8 human colorectal cancer cells. Hepatogastroenterology 58: 1951-1955, 2011.

26. Zuo W and Li H: Relationship of the expression of CD44v6 and paxillin to the prognosis of non-small cell lung carcinoma. Sichuan Da Xue Xue Bao Yi Xue Ban 34: 484-485, 2003 (In Chinese). 